\title{
Solution of population balance equations by logarithmic shape preserving interpolation on finite elements
}

\author{
Liborio I. Costa \\ Etzbergstrasse 19c, 8405, Winterthur, Switzerland \\ Giuseppe Storti \\ ETH Zurich, Vladimir-Prelog-Weg 1, 8093, Zurich, Switzerland \\ Stefano Lazzari \\ Massachusetts Institute of Technology, 77, Massachusetts Avenue, 02139 Cambridge, \\ $M A, U S A$
}

\begin{abstract}
A new numerical approach for solving population balance equations (PBE) is proposed and validated. The method employs a combination of basis functions, defined on finite elements, to approximate the sought distribution function. Similarly to other methods of the same family, the PBE are solved only in a finite number of values of the internal coordinate (grid points). The peculiarity of the method is the use of a logarithmic, shape-preserving interpolation (LSPI) procedure to estimate the values of the distribution in between grid points. The main advantages of the LSPI method compared to other approaches of the same category are: i) the stability of the numerical approach (i.e. the absence of oscillations in the distribution function occurring when using "standard" cubic splines and a low number of elements), and ii) the conceptual and implementation simplicity, as no mathematical manipulation of the PBE is required.
\end{abstract}

Keywords: Population balance equations, Aggregation, Breakage, Finite elements, Interpolation 


\section{Introduction}

The synthesis of new materials and the continuous technological progress allowed an impressive advancement in different fields, leading to the realization of new types of drugs, more efficient solar panels, electronics and plastics derived from renewable resources. Most of these materials are synthesized through an assembly process of subunits (molecules or particles) that typically form differently sized assemblies, rather than one-sized structures.[1, $2,3,4,5,6]$ To properly control and optimize these assembly processes, a mathematical framework is of indisputable importance. For this reason, the differential equation set, known as population balance equations (PBE), has been used extensively since its formal introduction.[7, 8]

PBE have been employed to rationalize polymerization reactions $[9,10$, $11,12,13,14,15,16,17,18,19,20,21]$, colloid aggregation, [22, 23, 3, 24, $25,26,27,28]$ and crystallization processes $[29,30,31,32,33]$. This short list proves the versatility of the PBE, that enable the description of virtually any type of population, focusing on some key property such as the number of monomeric units in polymer chains, or the size of crystals. These properties, usually referred to as the internal coordinates of the PBE, define the discrete (e.g. the number of monomers) or continuous nature (e.g. the length of a crystal) of the PBE.

Whether discrete or continuous, the PBE can be solved either with stochastic or deterministic approaches.[8] Stochastic methods are indeed very interesting and may reveal structural and topological information of the assemblies, but their lengthy computational times make them unsuitable to be employed for process optimization.[18, 8] Deterministic approaches overcome this hurdle and allow the quantification of kinetic rates. An overview of the available deterministic strategies to solve the PBE is discussed in the following, considering a one dimensional distribution function in its continuous formulation. These considerations equally apply to discrete balances and multidimensional PBE, although the latter remain out of the scope of the present paper. For this general considerations, we will refer to $f(x, t) d x$, representing the number concentration of species (i.e. clusters, chains, aggregates), consisting of $x$ to $x+d x$ units.

The first class of methods to be historically proposed, is based on the integral properties of the distribution, the so called "moments".[7] By focusing only on the moments of the distribution, the method delivers results by integrating only a few ordinary differential equations. The price to pay is 
threefold: i) the need of assuming a distribution shape in order to reconstruct the size distribution $f(x, t)$, ii) the lack of generality (some mass-dependent rate equations cannot be easily employed), and iii) the necessity of often having to employ closure equations. [34, 35] Nevertheless, the method of moments has been proven very powerful in dealing with multidimensional and complex balances, for instance in the case of non-linear polymerizations.[11, 18]

An improvement of the method of moments is represented by the quadrature method of moments (QMOM) and its variations.[28, 35] By solving a few more differential equations (usually 6-12), these approaches still suffer of the necessity of assuming a distribution shape when reconstructing $f(x, t)$, but overcome the remaining issues of the method of moments. Providing average properties accurately and with little computational power, enabled the method of moments and the QMOM to solve PBE in spatially distributed systems[36] and in computational fluid dynamic frameworks.[28, 35]

When interested in obtaining the full distribution $f(x, t)$ without making assumptions regarding its shape, other approaches such as i) the method of generating functions, ii) finite differences schemes, iii) the method of discretized PBEs, and iv) the method of basis functions can be used.

The generating function method $[37,38]$ relies on solving the PBE in the Laplace space and then inverting the solution to re-obtain the desired distribution. This inversion step is numerical in most cases and represents the main drawback of the generating functions approach, given the related numerical complexities. [9]

In the "finite differences" approach, both the time and the internal coordinate domain are discretized, replacing all derivatives in the PBE with finite differences. To this class belong also the high resolution finite volume methods, that employ higher-order approximation of the derivatives. These latter methods reduce the numerical diffusion problems often encountered by "standard" finite difference approaches in nucleation and growth problems. [39, 40] Nevertheless, these methods are computationally demanding when aggregation and breakage are of interest, because of the quite fine grids usually required to obtain accurate results. [41]

Discretized PBE methods, or method of classes or sectional methods, rely on the subdivision of the internal coordinate in a number of finite, continuous, intervals (or bins). [42, 43, 44, 15, 45] The representative values of the internal coordinate within each bin are referred to as pivotal values. The PBE are rewritten accounting for each pivotal value, imposing the preservation of one or more properties of the distribution. [42, 46] For example, whenever a cluster is 
formed whose mass does not correspond to a pivotal value, it is redistributed among the nearest pivots in order to preserve the first two moments of the distribution. The discretized PBE are one of the most applied methods, especially since the formulation due to Kumar and Ramkrishna.[42] Despite the constant improvement throughout the years, [46, 47, 45] the method may suffer of some inaccuracies in predicting higher order moments when a low number of pivots is used, and it is not of straightforward use because of the rather complex mathematical treatment necessary to implement and apply the methodology.

Another class of methods approximates the unknown distribution $f(x, t)$ using a set of known basis functions $\phi_{i}(x)$ multiplied by weighting coefficients $a_{i}(t)$, where $i=1,2, \ldots N$, with $N$ being the total number of basis functions employed. Within this category different sub-methods can be distinguished, according to a) the domain in which the basis functions $\phi_{i}(x)$ are defined, b) the specific form of the selected basis functions $\phi_{i}(x)$, and $\left.c\right)$ the way the coefficients $a_{i}(t)$ are determined. When the basis functions $\phi_{i}(x)$ are defined on intervals (e.g. when piecewise polynomials are used to approximate $f(x, t)$ ) one speaks of basis function on finite elements, $[48,49,50]$ otherwise, one refers to spectral basis functions. [51, 52] The choice of the basis function is key in this method, as it determines its stability and accuracy.[49] Many different functions have been tested to this end, ranging from linear functions, [49] to different cubic polynomials, [48, 53, 43] or Gaussian functions.[52] A further distinction among these methods is related to how the unknown coefficients (or weights) $a_{i}(t)$ are determined. [54] The "method of the weighted residuals" is one of the most popular for determining such coefficients.[48, 53, 51, 54] Here, the approximated form of the solution is substituted in the PBE thus giving a formulation of the residual function, which needs to be minimized. Depending on the minimization strategy, one obtains different formulations of the weighted residual method, such as the Galerkin or the collocation method. In a few other cases, no minimization of the residual is performed, and the coefficients are obtained "directly" by using $C^{2}$ cubic splines, [55] by solving an algebraic[48] or a differential equation system.[52]

In this frame, the present work introduces a new numerical approach that falls in the category of the basis functions method on finite elements (i.e. the basis functions are defined on intervals). Instead of considering $f(x, t)$, we approximate its natural logarithm, $\ln (f(x, t))$, using a cubic polynomial: $\ln (f(x, t)) \approx a_{i}(t) x^{3}+b_{i}(t) x^{2}+c_{i}(t) x+d_{i}(t)$, where $i$ represents the $i^{\text {th }}$ interval considered. This implies that the $f(x, t)$ is approximated in each 
interval $i$ with an exponential function: $f(x, t) \approx e^{a_{i}(t) x^{3}+b_{i}(t) x^{2}+c_{i}(t) x+d_{i}(t)}$. Notably, Hermite shape-preserving cubics are considered to approximate the $\ln (f(x, t))$, implying that the determination of the time-dependent coefficients $\left(a_{i}, b_{i}, c_{i}, d_{i}\right)$ is direct and bypasses the calculation of a residual. The main advantages of the logarithmic shape preserving interpolation (LSPI) method are i) that no mathematical manipulation of the original PBEs is required, resulting in a simple numerical implementation when compared to other approaches, $[54,42,40]$ and ii) the stability and accuracy of the method even when using a low number of elements compared to similar approaches relying on low-order polynomials. [48, 49] By using a shape preserving interpolation, in fact, the oscillations often encountered when applying polynomial interpolations on sparse grids [56] are automatically avoided.

The work is structured as follows. First the LSPI method is presented and then validated employing aggregation and breakage mechanisms, using both discrete and continuous PBE. Initially, a purely aggregating system is considered and the LSPI predictions are compared with analytical solutions of the PBE using three different kernels (constant, sum, and product). Then a comparison with an accepted literature approach relying on Gaussian basis functions,[52] is carried out to validate the LSPI approach with diffusion-limited, and reaction-limited aggregation. Finally, the LSPI method is validated in the frame of aggregating-breaking systems. To this end, a random breakage kernel resulting in an analytical solution was employed. To test the LSPI approach also with non-analytical breakage problems, mass- and position-dependent breakage kernels have been selected and the LSPI solutions were compared with the aforementioned literature numerical approach.[52]

\section{Logarithmic shape-preserving interpolation - numerical and math- ematical aspects}

The logarithmic shape-preserving interpolation (LSPI) method is presented in this section. For the sake of clarity, the method is introduced for systems undergoing aggregation only, for both the continuous (Section 2.1) and the discrete (Section 2.2) formulations of the PBE. The straightforward generalization to the case of aggregation/breakage is shortly discussed in Section 3, while a complete list of symbols is provided in the appendix in Tables 4 and 5 . 


\subsection{Logarithmic shape-preserving interpolation - continuous PBE}

To illustrate the method, let us first consider the PBE for a system undergoing aggregation only:

$$
\begin{aligned}
\frac{\partial f(x, t)}{\partial t}= & -f(x, t) \int_{0}^{\infty} \beta(x, y) f(y, t) d y \\
& +\int_{0}^{x / 2} \beta(x-y, y) f(x-y, t) f(y, t) d y
\end{aligned}
$$

where $f(x, t)$ is the population density (or distribution function), and $f(x, t) d x$ represents the number concentration of clusters at time $t$ with size comprised between $x$ and $x+d x . \beta(x, y)$ is the aggregation kernel, describing the rate of combination of two clusters, of sizes $x$ and $y$. Note that $x$ and $y$ will be used along the text to indicate the sizes or masses of the clusters, hence they do refer to the same internal coordinate of the distribution function. The first term in equation 1 represents the rate of disappearance of the clusters of size $x$ due to their aggregation with other clusters (of any size). The second term, i.e. the convolution integral, represents the formation rate of an aggregate of size $x$ from the aggregation of smaller clusters.

The LSPI approach provides a solution for the PBE (equation 1) employing a 4-step procedure:

(i) The continuous PBE is written for a number $I$ of grid points $x_{i}$, where $i=1,2, \ldots, I$, obtaining the corresponding $I$ ordinary differential equations:

$$
\begin{aligned}
\frac{d f\left(x_{i}, t\right)}{d t}= & -f\left(x_{i}, t\right) \int_{x_{\min }}^{x_{\max }} \beta\left(x_{i}, y\right) f(y, t) d y \\
& +\int_{x_{\min }}^{x_{i} / 2} \beta\left(x_{i}-y, y\right) f\left(x_{i}-y, t\right) f(y, t) d y
\end{aligned}
$$

Here, $x_{1}=x_{\min }$ and $x_{I}=x_{\max }$ represent the minimum and maximum values of the internal coordinate used for the numerical solution. Note that the $I$ grid points $x_{i}$ define the boundaries of the $I-1$ finite elements in which the domain of $x$ is subdivided.

(ii) Each distribution function appearing in the integrals is interpolated on $J$ knots $y_{j}$, with $j=1,2, \ldots, J$, covering the full integration domain. With reference to equation 2 , this corresponds to obtaining the values 
$f\left(y_{j}, t\right)$ with $y_{1}=x_{\min }$ and $y_{J}=x_{\max }$ for the first integral, as well as $f\left(x_{i}-y_{j}, t\right)$ and $f\left(y_{j}, t\right)$ with $y_{1}=x_{\min }$ and $y_{J}=x_{i} / 2$ for the convolution integral.

(iii) The integrals in equation 2 are numerically evaluated using the $J$ knots of point ii), relying on standard quadrature approaches.

(iv) The $I$ time derivatives of $f\left(x_{i}, t\right)$ in equation 2 are calculated, and a time integration step is performed. Steps ii) and iii) are then repeated iteratively at each integration time step.

In the following, the two key steps of the LSPI method, interpolation and numerical integration, will be discussed, highlighting the differences of the LSPI approach compared to other methods using basis functions on finite elements.

\subsubsection{Interpolation}

In the context of PBE, the main requirements for an effective interpolant are:

(i) it approximates well the shape of the function $f(x, t)$ over the whole domain of the independent variable $x$;

(ii) it can be computed easily and efficiently;

(iii) it preserves the non-negativity of $f(x, t)$;

(iv) it requires a low number of interpolating points.

Hermite shape preserving cubic polynomials fulfill all the above requirements and do not need any a-priori knowledge of the shape of the function to be interpolated.[57, 58] Moreover, they are relatively straightforward to compute.

Unlike other methods which use (non-shape preserving) $C^{1}$ cubic interpolants, they do not require the definition of additional points (collocation points) within each finite elements where the residual of the differential equation has to be minimized in order to determine the coefficients of the interpolant.[48, 53, 58, 27] In our case, the cubic coefficients are obtained directly from a step by step approach once the coordinates $\left(x_{i}, f\left(x_{i}, t\right)\right)$ are known without any need to solve for large algebraic or algebraic-differential systems. Additionally, they are also free from the oscillations and overshootings that may arise when using $C^{2}$ cubic splines and only a few interpolation points.[58, 56]

Recalling that at each time $t$ we know the values of the distribution function in the $I$ grid points $x_{i}$, using a shape preserving Hermite polynomial 
implies that $(I-1)$ cubic interpolants $P(y, t)$ will be used, one for each of the $(I-1)$ elements.

In order to limit the number of elements required to approximate $f(x, t)$, it is useful recalling that for aggregating systems the population density $f(x, t)$ is often of exponential nature $[48,59]$. Therefore, one can infer that the same interpolation accuracy may be achieved with fewer grid points when interpolating the logarithm of the density function rather than the density function itself. In other words, instead of using $f\left(x_{i}, t\right)$, we will use the values $\ln \left(f\left(x_{i}, t\right)\right)$ to carry out the interpolation.

Employing the $\ln \left(f\left(x_{i}, t\right)\right)$ comes with a caveat. Although the population density is non-negative in the whole domain by definition, numerical errors may arise when integrating equation 2, as in any other approximated approach, leading the $f\left(x_{i}, t\right)$ values to be negative in some points, thus precluding the logarithmic transformation. This may occur during the time integration when the population decays rapidly and has extremely low values. Therefore, we define a local shift factor $\epsilon$ :

$$
\epsilon=\left(1+d_{\epsilon}\right)\left|\min \left\{f\left(x_{i}, t\right) \backslash\{0\}\right\}\right|
$$

with $d_{\epsilon}>0$. The local shift factor $\epsilon$ is a positive number whose value is larger (by a factor $\left(1+d_{\epsilon}\right)$ ) than the modulus of the minimum of the non-zero elements of $f\left(x_{i}, t\right)$. Accordingly, whatever the values of the population density at the grid points, $f\left(x_{i}, t\right)+\epsilon$ is always positive, and one can safely apply the shape preserving cubic splines using the known coordinates $\left(x_{i}, \ln \left(f\left(x_{i}, t\right)+\epsilon\right)\right)$, from which the piecewise cubic interpolant $P(y, t)$ can easily be obtained. With this in mind the sought interpolating functions, satisfying the above mentioned conditions and referring to the interval $h_{i}=\left[x_{i} \leq y<x_{i+1}\right]$, will have the form:

$$
\begin{aligned}
P(y, t)= & \ln \left(f\left(x_{i}, t\right)+\epsilon\right)+\left(y-x_{i}\right) d_{i} \\
& +\left(y-x_{i}\right)^{2} c_{i}+\left(y-x_{i}\right)^{3} b_{i} \\
& \text { where } x_{i} \leq y<x_{i+1} \\
& \text { and } i=1,2, \ldots, I-1
\end{aligned}
$$

The parameters $b_{i}, c_{i}$ and $d_{i}$ required to obtain the shape-preserving interpolant are obtained through a well-established procedure by Fritsch and Carlson, [58, 57] reported in the ESI (Section S1). 
Equation 4 gives access to the value of the population density $f(y, t)$ at a desired coordinate $y$ by a simple inversion:

$$
f(y, t)=\exp (P(y, t))-\epsilon
$$

A synoptic view of the interpolation procedure is illustrated in Figure 1. From the known coordinates at time $t, f\left(x_{i}, t\right)$, one determines the minimum of the non-zero $f\left(x_{i}, t\right)$ values (equation 3 and Figure 1a), from which the shift factor $\epsilon$ is calculated (equation 3). This allows the calculation of $\ln \left(f\left(x_{i}, t\right)+\right.$ $\epsilon)$. Then the coefficients of the cubic interpolant $\left(b_{i}, c_{i}, d_{i}\right)$ are determined in interval $h_{i}$ (ESI, Section S1) for the set of points $\left(x_{i}, \ln \left(f\left(x_{i}, t\right)+\epsilon\right)\right)$, as shown in Figure 1b). This interpolation procedure is repeated for all the $I-1$ intervals, hence the logarithm of the shifted function can be evaluated at any value $y_{j}$ (equation 4 and Figure 1c)). Finally, the density function itself is obtained through equation 5 (Figure 1d)).

\subsubsection{Quadrature}

After having interpolated using the known $I$ points and having calculated the distribution function in all selected knots, it is possible to numerically evaluate the integrals in equation 2. Since numerical integration methods are well-known, [60] we report the corresponding details in the ESI (Section S2), while the most relevant observations on the quadrature method used are summarized here:

(i) the coordinates of the $J$ knots for the quadrature are defined independently from the $I$ grid points $x_{i}$, decoupling the number of ordinary differential equations to be solved $(I)$, from the appropriate number of points to perform the numerical integration;

(ii) The same number of $J$ knots is used for the quadrature of all the PBE integrals (equation 2). Although not strictly necessary, this choice limits to a minimum the parameters of the method;

(iii) Logarithmic abscissas and higher order quadrature rules lead to a better accuracy than linear abscissas or simple trapezoidal integration (ESI, Section S2). Nevertheless, all quadrature methods converge to the same results when using a large enough number of interpolating knots, $J \approx 10^{2}$, confirming that any quadrature approach could be used. We used the $4^{\text {th }}$ degree Newton-Cotes formulas (Boole's rule)[60] using a logarithmic scale for the integration variable, because it provided high accuracy with limited knots number (ESI, Section S2). 

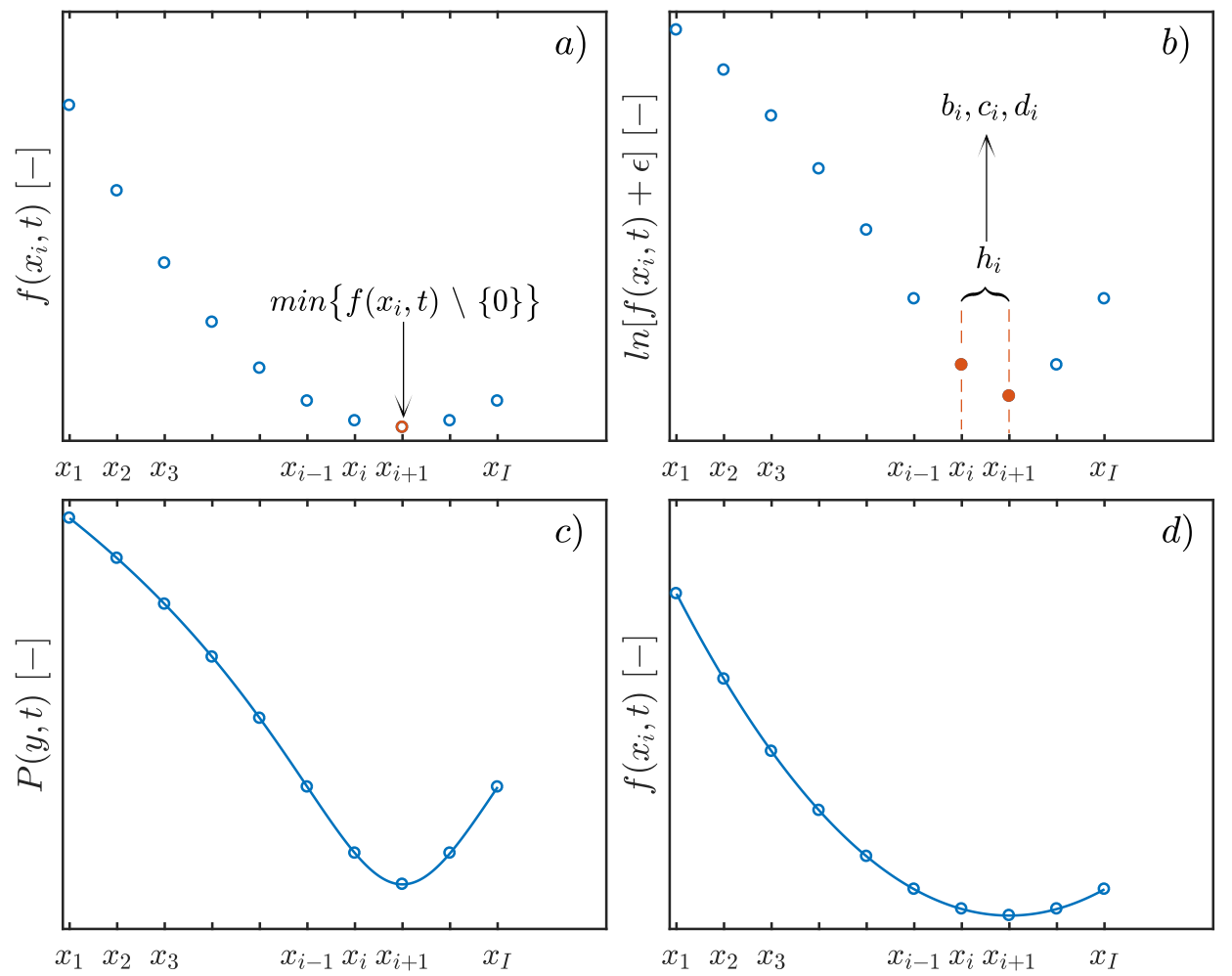

Figure 1: Scheme illustrating the interpolation procedure. In particular: a) find the minimum of $f\left(x_{i}, t\right)$, and compute $\epsilon$ with equation 3 ; b) compute $\ln \left(f\left(x_{i}, t\right)+\epsilon\right)$ and the coefficients of the shape preserving cubic spline $\left(b_{i}, c_{i}, d_{i}\right)$ in the interval $h_{i}$ (equation 4 and ESI Section S1), repeat for all intervals $I-1 ;$ c) use the obtained coefficients and the interpolant function $P(y, t)$ to estimate the function at selected $y$ values (equation $4 ; \mathrm{d}$ ) convert the found values $P(y, t)$ to $f(y, t)$ using equation 5 . 
286

\subsection{Logarithmic interpolation - discrete PBE}

In the case of discrete PBE, the balance for an aggregating system becomes:

$$
\begin{aligned}
\frac{d f(x, t)}{d t}= & -f(x, t) \sum_{y=1}^{\infty} \beta(x, y) f(y, t) \\
& +\frac{1}{2} \sum_{y=1}^{x-1} \beta(x-y, y) f(x-y, t) f(x, t)
\end{aligned}
$$

Here $f(x, t)$ denotes the number concentration of the clusters with size $x$. Note that, in the case of discrete populations, $f(x, t)$ does not represent a density distribution, but the number (or concentration) of species with internal coordinate $x \in \mathbb{N}$, and $f(x, t)$ is defined only on the points $x \in \mathbb{N}$. To use the LSPI approach also for discrete PBEs, we write the PBEs for a finite number $I$ of grid points $x_{i}$, and replace the summations with integrals using the Euler-Maclaurin approximation. Combining these two steps, one gets:

$$
\begin{aligned}
\frac{d f\left(x_{i}, t\right)}{d t} \approx & -f\left(x_{i}, t\right)\left[\frac{m_{i}(1)}{2}+\int_{1}^{x_{\max }} m_{i}(y) d y+\frac{m_{i}(J)}{2}\right] \\
& +\left[\frac{n_{i}(1)}{2}+\int_{1}^{\left\lfloor x_{i} / 2\right\rfloor} n_{i}(y) d y+\theta_{x_{i}} \frac{n_{i}\left(y_{J}\right)}{2}\right]
\end{aligned}
$$

The integrand functions $m_{i}(y)$ and $n_{i}(y)$ are continuous functions approximating the sequences to be summed up in equation 6 , for which the timedependency has been dropped for the sake of brevity:

$$
m_{i}(y)=\beta\left(x_{i}, y\right) f(y, t)
$$

$$
n_{i}(y)=\beta\left(x_{i}-y, y\right) f\left(x_{i}-y, t\right) f(y, t)
$$

$\left\lfloor x_{i} / 2\right\rfloor$ represents the floor of $x_{i} / 2$ and $\theta_{x_{i}}=x_{i} \bmod 2$ :

$$
\theta_{x_{i}}= \begin{cases}1, & \text { if } x_{i} \text { is odd } \\ 0, & \text { if } x_{i} \text { is even }\end{cases}
$$

Note that equation 7 implies that the summations in equation 6 are replaced by integrals plus two terms for each summation which add half of the extreme 
values of the integrals, according to the Euler-Maclaurin approximation. In other words, although in the discrete case $f(x, t)$ has physical meaning only on the points $x_{i} \in \mathbb{N}$, by means of the Euler-Maclaurin approximation we can solve the discrete PBE in equation 6 by applying the very same methodology used for solving the continuous PBE to equation 7 . The integrand functions are evaluated on $J$ knots by interpolation of the $I$ known distribution values $f\left(x_{i}, t\right)$, and the integrals are numerically evaluated. In the following, test cases are illustrated to validate the method.

\section{Validation}

In the present section the LSPI method is validated in different scenarios. The results are reported using the non-dimensional time $\tau$ defined as: $[48,59]$

$$
\tau=\beta(1,1) \mu_{0}(t=0) t
$$

where $\mu_{0}(t=0)=\int_{0}^{\infty} f(x, t=0) d x$ is the moment of order 0 , i.e. the total particle concentration, at time zero, and $\beta(1,1)$ is the rate of aggregation of two primary particles. The LSPI method was implemented in Matlab and the PBEs were solved on a PC with 6 cores and processor AMD Phenom $2.7 \mathrm{GHz}$. The system of ordinary differential equations was integrated using standard built-in ODE solvers. Unless otherwise stated, $d_{\epsilon}=10^{-3}$ (equation 3 ) and an explicit Runge-Kutta $(4,5)$ solver with a relative tolerance of $10^{-6}$ and absolute tolerance of $10^{-20}$ were used.

\subsection{Continuous PBE - constant and sum kernel}

Initially, the LSPI approach is validated using continuous PBE in the frame of an aggregation problem using the constant and sum kernels. In these scenarios, analytical solutions of the PBE were available and could be compared with the numerical results of the LSPI method. The employed parameter values are reported in Table 1, and the results in Figure 2.

In Figure 2, the continuous black lines represent the analytical solution, the empty red circles the $I$ grid points employed (i.e. the number of solved equations), and the red dashed lines the interpolated numerical solution. Panel a) shows a very good superimposition between numerical and analytical distributions when employing the constant kernel. Notably, panel b) reports the very same distributions as in a), plotted on a logarithmic axis in order to appreciate the performance of the numerical solution, that overlaps with the 


\begin{tabular}{lll}
\hline Parameters & Constant kernel & Sum kernel \\
\hline$\beta(x, y)$ & 1 & $(x+y)$ \\
{$\left[x_{\text {min }}, x_{\text {max }}\right]$} & {$\left[10^{-3}, 500\right]$} & {$\left[10^{-3}, 10^{4}\right]$} \\
Initial condition & $f(x, 0)=\frac{N_{0}}{x_{o}} e^{-\frac{x}{x_{0}}}$ & $f(x, 0)=\frac{N_{0}}{x_{o}} e^{-\frac{x}{x_{0}}}$ \\
Simulated $\tau[-]$ & {$[0-10]$} & {$[0-4]$} \\
$I[-]$ & 3 & 15 \\
$J[-]$ & 81 & 81 \\
\hline
\end{tabular}

Table 1: Kernel and parameters for the solution of continuous PBE, Figure 2. For the initial conditions, $N_{0}=x_{0}=1$ were used. Analytical solutions are available in [48]
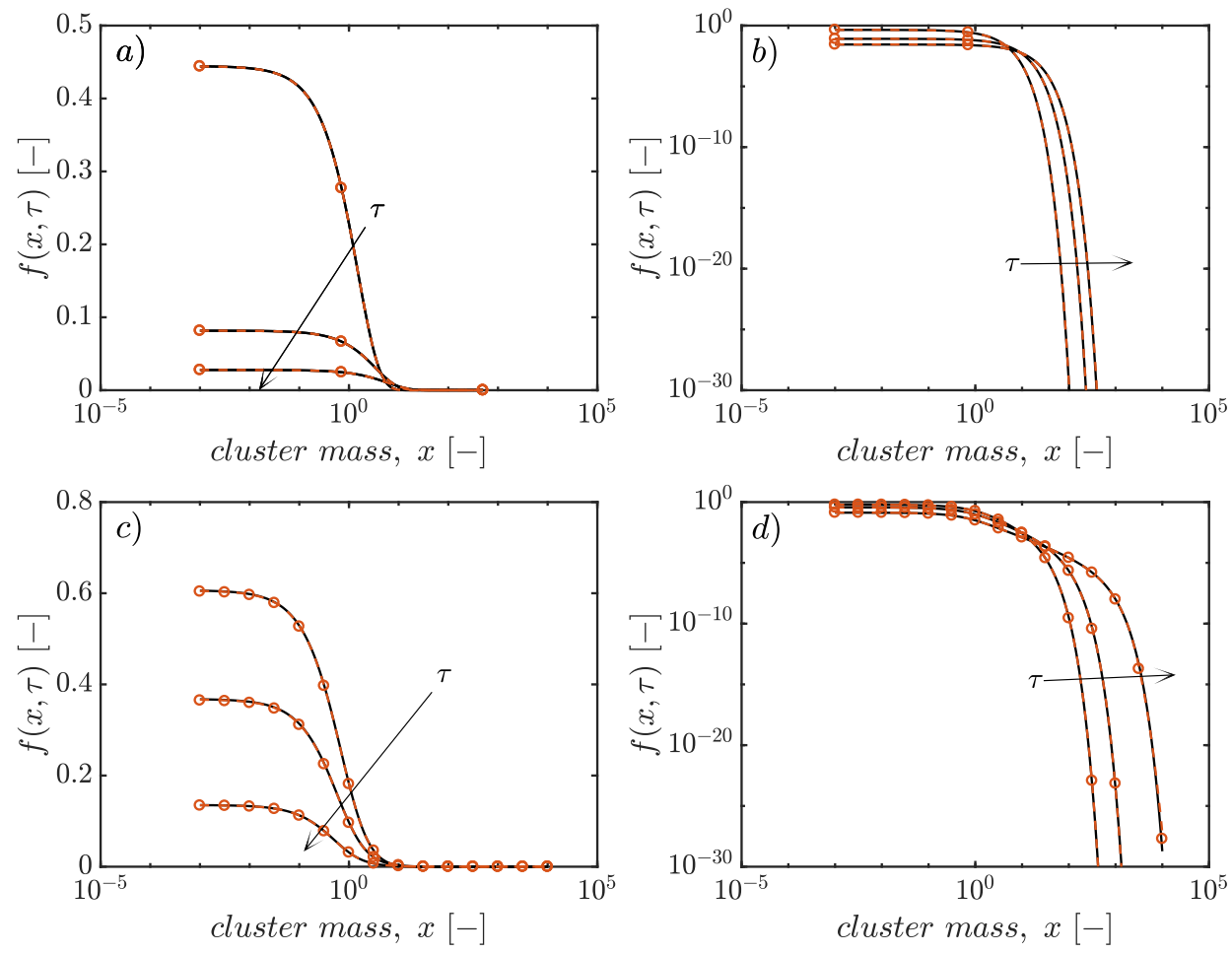

Figure 2: Time evolution of the population density for pure aggregation: LSPI solution (red) and analytical solution (black). Constant kernel, with a) linear and b) logarithmic representation of the $y$-axis (at $\tau=1,5,10$ ). Sum kernel, with c) linear and d) logarithmic representation of the $y$-axis (at $\tau=1,2,4$ ). 
analytical one for 30 orders of magnitude (odm) on the y-axis and covering 6 odm on the x-axis. Similar considerations hold when inspecting Figure 2 c) and d), that show distributions obtained with the sum kernel. Once again, a very good overlap of the numerical and analytical solution is observed both when plotting the results on a linear (panel c)) and logarithmic axis (panel d)). The LSPI approach guarantees in both cases highly accurate solutions using a very low number of grid points and, therefore, of differential equations to be solved. In particular, $I=3$ for the constant kernel and $I=15$ for the sum kernel, while in both cases $J=81$ interpolating knots were used to compute the integrals in equation 2 . The very low number of grid points used with the constant kernel is consequential to the logarithmic interpolation strategy: starting with an exponential initial condition, the exponential profile is preserved as the time increases and only three grid points (and thus three differential equations) suffice to interpolate $f(x, t)$ (Figure 2 a) and b)).

We have further proven that the logarithmic interpolation strategy is key for obtaining accurate results even when using very few elements. This is shown for the sum kernel in the ESI (Figure S4 a) and b)) where solutions obtained with and without employing the logarithm of the distribution function for the interpolation are shown. While the LSPI method superimposes with the analytical solution already with $I=15$, the same occurs with a "standard" shape-preserving interpolation (SPI) only when using $I=45$, and even then a deviation of the SPI is observed at the right tail of the distribution (cf. ESI, Figure S4b)).

\subsection{Discrete PBE - pure aggregation}

To test the LSPI approach in the case of discrete PBE, three further test cases have been considered (Table 2), using a) the product kernel, b) the diffusion-limited cluster aggregation (DLCA) kernel, and c) the reactionlimited cluster aggregation (RLCA) kernel. An analytical solution exists for the product kernel[59]. For the DLCA and RLCA kernels, the LSPI solution was compared against the numerical solution provided by the Gaussian basis functions (GBF) method.[52, 61, 62, 33]

In the discrete cases, the initial population distribution typically presents a singularity at $x_{i}=1$. In such a case, it is therefore preferable to define the $I$ grid points using a unitary spacing for the first few points, up to a given value $I_{c}$. Then, the usual exponential spacing can be used. In a similar way, the $J$ knots are defined on a unitary spaced grid up to $j=I_{c}$ and 


\begin{tabular}{llll}
\hline Parameters & Product kernel & DLCA kernel & RLCA kernel \\
\hline$\beta(x, y)$ & $2 x y$ & $\frac{2 k_{B} T}{3 \eta W W} B(x, y)$ & $\frac{2 k_{B} T}{3 \eta W} B(x, y) P(x, y)$ \\
$B(x, y)$ & - & $\left(x^{\frac{1}{d_{f}}}+y^{\frac{1}{d_{f}}}\right) \times$ & $\left(x^{\frac{1}{d_{f}}}+y^{\frac{1}{d_{f}}}\right) \times$ \\
& - & $\left(x^{-\frac{1}{d_{f}}}+y^{-\frac{1}{d_{f}}}\right)$ & $\left(x^{-\frac{1}{d_{f}}}+y^{-\frac{1}{d_{f}}}\right)$ \\
$P(x, y)$ & - & - & $(x y)^{0.5}$ \\
{$\left[x_{m i n}, x_{\text {max }}\right]$} & {$\left[10^{0}, 5 \times 10^{6}\right]$} & {$\left[10^{0}, 5 \times 10^{5}\right]$} & {$\left[10^{0}, 5 \times 10^{5}\right]$} \\
$T[K]$ & - & 298.15 & 298.15 \\
$\eta[P a s]$ & - & $8.9 \times 10^{-4}$ & $8.9 \times 10^{-4}$ \\
$D_{p}[m]$ & - & $100 \times 10^{-9}$ & $100 \times 10^{-9}$ \\
$\phi[-]$ & - & $10^{-4}$ & $10^{-4}$ \\
$W[-]$ & - & 1 & $10^{4}$ \\
$d_{f}[-]$ & - & 1.8 & 2.1 \\
I.C. $f(1,0)$ & 1 & $\frac{6 \phi}{\pi D_{p}^{3}}$ & $\frac{6 \phi}{\pi D_{p}^{3}}$ \\
Simulated $t[s]$ & - & $4200 s$ & $42000 s$ \\
Simulated $\tau[-]$ & 0.998 & - & - \\
$I_{c}[-]$ & 10 & 10 & 10 \\
$I[-]$ & 26 & 26 & 26 \\
$J[-]$ & 90 & 90 & 90 \\
\hline
\end{tabular}

Table 2: Kernel and parameters for the solution of discrete PBE, Figure 3 and Figure 4. The absolute tolerance was $10^{-50}$ when using the product kernel, and $10^{-20}$ for the DLCA and RLCA kernels. The analytical solution for the product kernel is from reference [59]. Note that $f(x>1,0)=0 \forall x>1$. I indicates the total number of grid points, including $I_{c}$. 

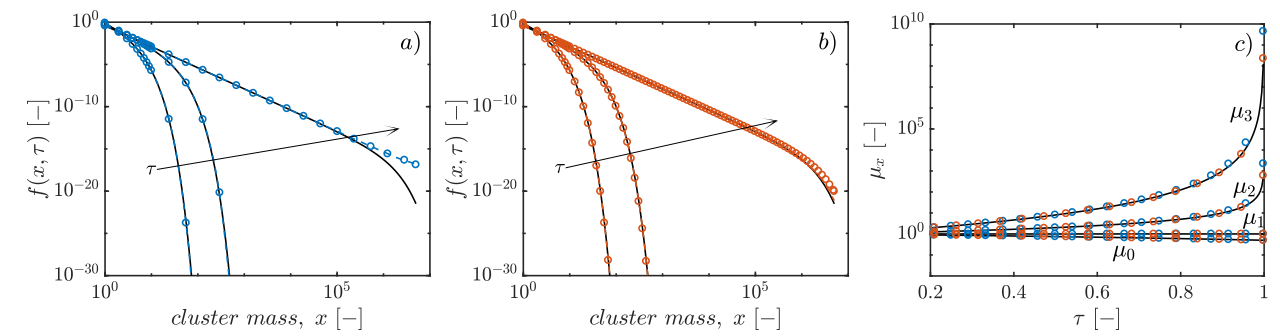

Figure 3: Discrete PBE solved for pure aggregation using the product kernel. a) and b) Distribution $f(x, \tau)$ for $\tau=\{0.2,0.6,0.998\}, \mathrm{c})$ moments of order $0^{\text {th }}-3^{\text {rd }}$ vs. $\tau$. The analytic solutions [59] are displayed using black continuous lines. Dashed blue (with $I=26$ and $J=90,(\mathrm{a}))$ and red $(I=80$ and $J=90(\mathrm{~b}))$ lines are the interpolated distributions, obtained from the distribution computed at the $I$ grid point (circles).

on an exponentially spaced grid (uniform on the logarithmic scale) until the upper integration limit. Accordingly, the Euler-McLaurin approximation is applied after the first $I_{c}-1$ terms of the summations in Equation 6, which are summed directly. The numerical integration using Boole's rule on logarithmic abscissa coordinates was applied from $y=I_{c}$ to the upper integration limit. Note that, given the discrete nature of the currently considered PBE, the $I$ grid points $x_{i}$ have to be natural numbers (equation 6 and 7 ), while this limitation is not required for the $J$ knots.

\subsubsection{Product kernel - analytical solution}

The comparisons between the results provided by the LSPI numerical method and the analytical solution of the product kernel are reported in Figure 3.

In particular, Figure 3 a) shows the performance of the LSPI method using $I=26$ grid points and $J=90$ interpolation knots. A good overlap between analytical and numerical solution is observed for the distributions at lower times, but a slight discrepancy arises for the largest $\tau$ (Figure 3a). This discrepancy vanishes as soon as the number of grid points is increased to $I=80$, as shown in Figure 3b. It should be noted that the aforementioned discrepancy was observed at normalized concentrations as low as $10^{-20}$, and only in close proximity of the gelation point,[18] $(\tau=1)$, while the agreement with the analytical solution was otherwise very good for the entire simulated time. To further prove this point the moments of order 0 to $3\left(\mu_{0}, \mu_{1}, \mu_{2}\right.$ and $\left.\mu_{3}\right)$ are reported against time in $3 \mathrm{c}$ ). In particular, the blue circles 
represent the moments computed using $I=26$ grid points, the red circles the solution with $I=80$, and the continuous black lines the analytical solution. A discrepancy between the analytical solution and the LSPI method with $I=26$ is observed only for the higher order moments at times close to the gelation of the system (typically identified when the $2^{\text {nd }}$ and higher order moments diverge).[18] This further proves that even when using a very low number of grid points ( $I=26$ to simulate sizes up to $5 \times 10^{6}$ !), an accurate solution is actually obtained for practically the whole simulated time.

\subsubsection{DLCA and RLCA aggregation}

To verify whether the LSPI method works well also with non-analytical kernels, the DLCA and RLCA aggregation kernels have been considered. The LSPI predictions were compared with a validated literature method relying on Gaussian basis functions (GBF).[52, 61, 62] The parameter values employed for these calculations are reported in Table 2 and the results are displayed in Figure 4, where the dotted lines are the GBF solutions, while the red circles refer to the $I$ grid points of the LSPI method. Notably, a very good overlap can be observed over 5 orders of magnitude on the abscissas and over 15-20 orders of magnitude on the ordinates. When the concentrations are too low $\left(\approx 10^{0} \# / \mathrm{m}^{3}\right.$, given an initial particle concentration of $\left.\approx 10^{17} \# / \mathrm{m}^{3}\right)$ the GBF method starts exhibiting a 'nervousness', as already reported when the method was initially derived.[52] The LSPI method does not show any such instability, as intrinsically the method is designed to compute $f(x, t)$ between the grid points with a shape-preserving cubic spline (Section 2). To further prove that the two methods are indeed performing very similarly, and that the arising discrepancy is only due to a numerical problem arising at very low concentration, the first four moments $\left(0^{\text {th }}-3^{\text {rd }}\right)$ are reported against time for the two methods for DLCA in Figure 4 c) and for RLCA in Figure $4 \mathrm{~d}$ ). Once more, the LSPI method is represented by dashed red lines, while dotted blue lines have been employed for the GBF method. The very good overlap of the predictions, confirms that the LSPI approach is well-suited also to deal with complex kernels, such as those involved in the DLCA and RLCA aggregation mechanisms, by solving a small number of ODE. Notably, the logarithmic shape-preserving interpolation (LSPI) has been proven significantly better compared to its non-logarithmic counterpart, the shape-preserving interpolation (SPI) (ESI, Figure S4c). With the same amount of grid points $I=26$, only the LSPI solution overlaps with the reference one provided by the GBF method, while the SPI prediction does 

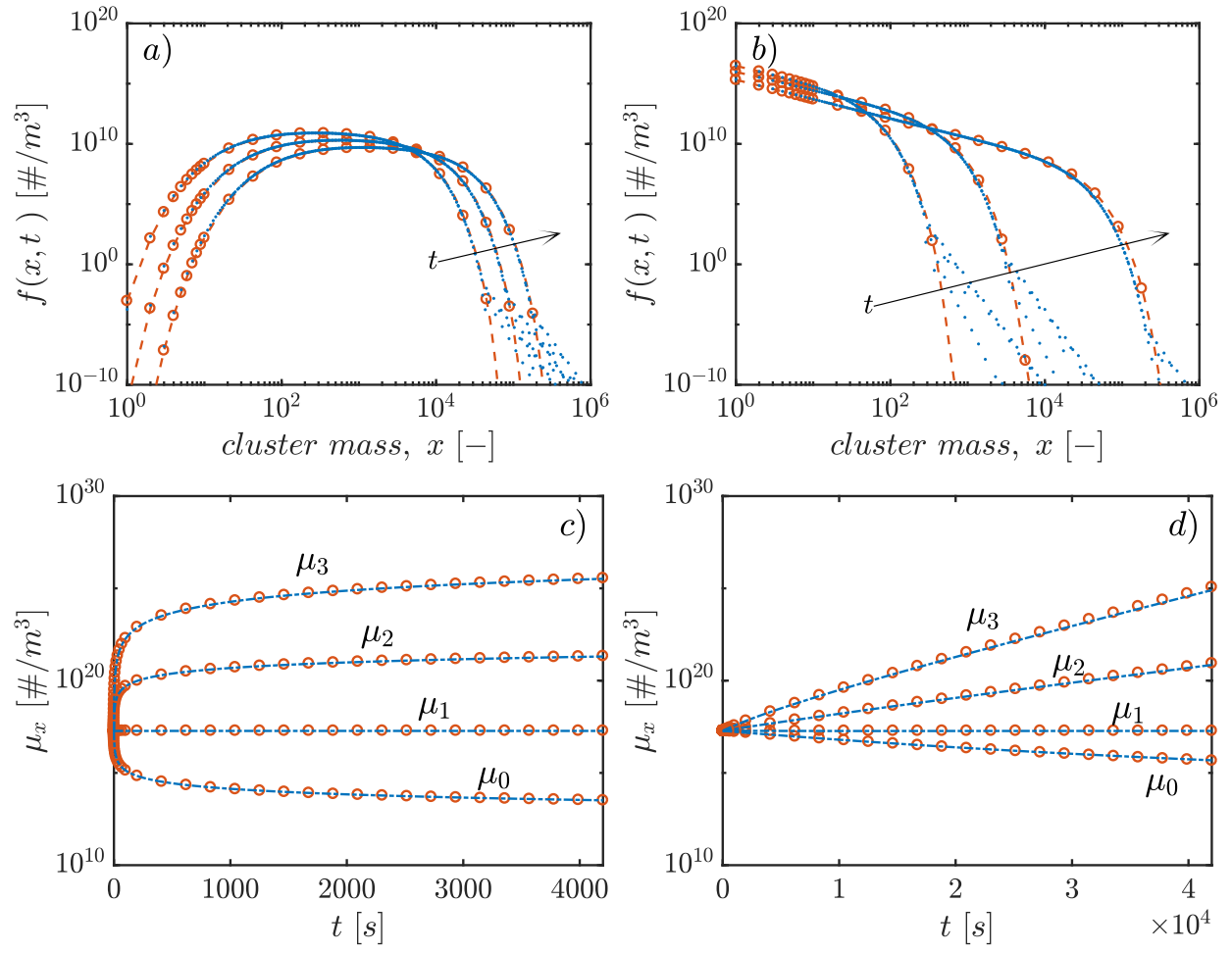

Figure 4: Discrete PBE for pure aggregation solved using a) the DLCA kernel at $\bar{t}=$ $\{1050 s, 2100 s, 4200 s\}$ and b) the RLCA kernel at $\bar{t}=\{10500 s, 21000 s, 42000 s\}$. The moments of order $0^{t h}-3^{r d}$ against time are reported for c) the DLCA case, and d) the RLCA case. The blue dotted lines were obtained using the Gaussian basis functions (GBF) method, the red dashed lines with the Logarithmic shape-preserving interpolation (LSPI) method. 
not (ESI, Figure S4c).

\subsection{Discrete PBE - Aggregation and Breakage}

To further prove the versatility of the proposed approach, aggregation and breakage problems have been considered as well. The general population balance accounting for aggregation and breakage reads:

$$
\begin{aligned}
\frac{d f(x, t)}{d t}= & -f(x, t) \sum_{y=1}^{\infty} \beta(x, y) f(y, t) \\
& +\frac{1}{2} \sum_{y=1}^{x-1} \beta(x-y, y) f(x-y, t) f(x, t) \\
& -\gamma(x) f(x, t)+2 \sum_{y=x+1}^{\infty} \Gamma(x, y) \gamma(y) f(y, t)
\end{aligned}
$$

Here $\gamma(x)$ represents the rate of breakage for the aggregate of size $x$, while $\Gamma(x, y)$ is the so-called daughter distribution function, representing the probability that an $x$-sized cluster forms from the breakage of a bigger cluster of size $y$. The cases considered in this work rely on a parabolic daughter distribution function: [44]

$$
\begin{aligned}
\Gamma(x, y)= & \frac{0.5 C}{y-1}+(1 / 2-C / 4)\left[\frac{8\left(3 x^{2}-3 x+1\right)}{(y-1)^{3}}\right. \\
& \left.-\frac{12(2 x-1)}{(y-1)^{2}}+\frac{6}{(y-1)}\right]
\end{aligned}
$$

As for the case of pure aggregation, to solve the PBE with the LSPI method, the summations in equation 13 are transformed into integrals by means of the Euler-Maclaurin approximation:

$$
\begin{aligned}
\frac{d f\left(x_{i}, t\right)}{d t}= & -f\left(x_{i}, t\right)\left[\frac{m_{i}(1)}{2}+\int_{1}^{x_{\max }} m_{i}(y) d y+\frac{m_{i}(J)}{2}\right] \\
& +\left[\frac{n_{i}(1)}{2}+\int_{1}^{\left\lfloor x_{i} / 2\right\rfloor} n_{i}(y) d y+\theta_{x_{i}} \frac{n_{i}\left(y_{J}\right)}{2}\right] \\
& -\gamma\left(x_{i}\right) f\left(x_{i}, t\right) \\
& +\left[\frac{p_{i}(1)}{2}+\int_{x_{i}+1}^{x_{\max }} p_{i}(y) d y+\frac{p_{i}(J)}{2}\right]
\end{aligned}
$$




\begin{tabular}{llll}
\hline Breakage Parameters & Random & Mass & Erosion \\
\hline$\beta(x, y)$ & 1 & $\left(x^{1 / 3}+y^{1 / 3}\right)^{3}$ & $\left(x^{1 / 3}+y^{1 / 3}\right)^{3}$ \\
$\gamma(x), \forall x>1$ & $10^{-4}(x-1)$ & $0.1 e^{0.01 x}$ & $2 x^{0.5}$ \\
$C$ & 2 & 2 & 0.5 \\
{$\left[x_{\text {min }}, x_{\text {max }}\right]$} & {$\left[1,5 \times 10^{3}\right]$} & {$\left[1,3 \times 10^{3}\right]$} & {$\left[1,2 \times 10^{3}\right]$} \\
Initial condition & $f(1,0)=1$ & $f(1,0)=1$ & $f(1,0)=1$ \\
& $f(x>1,0)=0$ & $f(x>1,0)=0$ & $f(x>1,0)=0$ \\
Simulated $\tau[-]$ & {$[0-5000]$} & {$[0-80]$} & {$[0-80]$} \\
$I_{C}[-]$ & 10 & 10 & 15 \\
$I[-]$ & 25 & 60 & 30 \\
$J[-]$ & 90 & 90 & 95 \\
\hline
\end{tabular}

Table 3: Note that $\gamma(x)$ is defined only for $x>1$, as primary particles are assumed not to break. A relative tolerance of $10^{-4}$ was used for all cases. The absolute tolerance was $10^{-20}$ for the random breakage and $10^{-15}$ otherwise. In all cases, the Matlab integrator "ode15s" has been used.I represent the total number of grid points including $I_{c}$. The analytical solution for random breakage [63] has been reported in ESI Section S3.

where $p_{i}(y)$ is the integrand function, for which the time-dependency has been dropped for the sake of compactness:

$$
p_{i}(y)=2 \Gamma\left(x_{i}, y\right) \gamma(y) f(y, t)
$$

The PBE in equation 14 were solved for three different cases, a random breakage kernel, a mass-dependent breakage, and a mass- and positiondependent breakage with parabolic daughter distribution. The corresponding kernels are reported in the ESI, Section S3, while the values of the kinetic parameters employed are shown in Table 3. The results obtained with the LSPI method were compared with analytical solutions (where available) or with the numerical solution obtained with the aforementioned Gaussian basis function approach.[52]

\subsubsection{Random breakage}

To test the LSPI approach in a simple breakage scenario, a random breakage mechanism has been employed. In particular, $\gamma(x)=10^{-4}(x-1)$, indicates that breakage may occur in any of the $x-1$ contact points with the same probability (assuming binary contact of primary particles). Note that the more complex product of $\Gamma(x, y) \gamma(y)$ simplifies to 1 by using $C=2$ and 

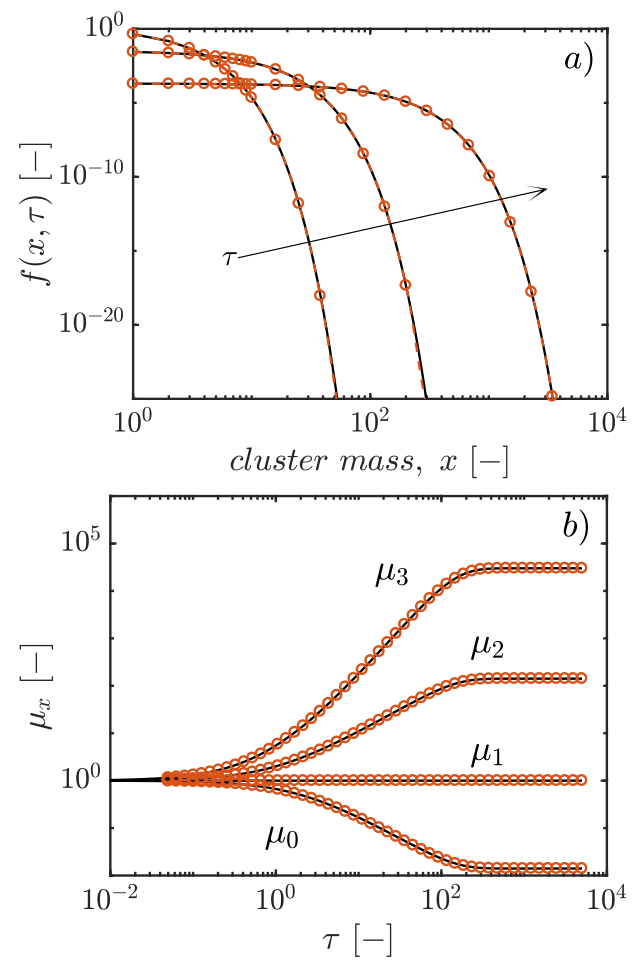

Figure 5: Discrete PBE for constant aggregation and breakage using the parameters reported in Table 3. Figure a) shows the distributions at non-dimensional times $\tau=1,10,5000$ and $\mathrm{b}$ ) the time-evolution of the moments of order $0^{t h}-3^{r d}$. The black lines represent the analytical solution [63], the red dashed lines and circles the results of the logarithmic shape-preserving interpolation (LSPI) method.

the above mentioned expression for $\gamma(y)$. For a constant aggregation mechanism and this simple breakage scheme an analytical solution exists.[63] Distributed and average properties obtained with the LSPI method (red dashed lines and circles) have been compared with the analytical solutions (black solid lines) in Figure 5.

A perfect overlap of all properties can be observed when inspecting Figure 5. Notably, $I=25$ nodes and $J=90$ knots were sufficient to obtain a very good overlap over more than 20 orders of magnitude for the distributions (Figure $5 \mathrm{a}$ )). The transition towards a stable stationary state where aggregation and breakage reach an equilibrium is well-captured by the LSPI method, as can be seen in Figure 5 b). 


\subsubsection{Mass- and position-dependent breakage}

To test the LSPI approach with more complex breakage kernels, two literature cases (Table 3) were selected.[44] The first considered kernel is only mass-dependent with $\gamma(x)=0.1 e^{0.01 x}$, i.e. larger clusters break faster than smaller ones. As in this first case, at $C=2$, no position dependency arises as $\Gamma(x, y)$ simplifies to 1 (equation 13 and ESI Section S3). The second kernel is instead a mass- and position-dependent breakage kernel. In this latter case larger clusters break easier than smaller ones (in fact $\gamma(x)=2 x^{0.5}$ ), and breakage occurs more likely in the so-called "erosion" mode. This results from using $C=0.5$ in equation 13 and implies that the most favored breakage events are those involving the "detachment" of smaller fragments (ESI, Section S3). Given that no analytical solution exists for the above mentioned kernels, a numerical comparison of the LSPI approach with the Gaussian basis function method has been performed. Both distributed (panels a) and b)) and average properties (panels c) and d)) have been considered (Figure $6)$.

A perfect overlap of the two methods is observed in all cases. Despite the more complex shape of the breakage kernels (Table 3 and ESI, Figures in Section S3), the LSPI method requires some more grid points only when dealing with the particularly stiff mass-dependent kernel. In any case, thanks to the decoupling between number of elements and quadrature knots, the number of grid points could be increased while keeping the same amount of interpolation knots as used in all other cases (Table 3). Moreover, it is worth mentioning that the Gaussian basis function method requires 180 bases (and a corresponding number of equations) in order to give satisfactory results. This is due to the shape of the base function: given that a very abrupt non-Gaussian distribution arises in the latter two cases, a high number of Gaussian bases are necessary to well-approximate the shape of the distribution. The LSPI method suffers less from this problem, as it is defined on finite elements, and because it deals with a shape-preserving interpolation of the logarithm of $f(x, t)$. As a result, it is less sensitive to the shape of the distribution and requires significantly less grid points and a corresponding number of differential equations to give comparable results in terms of system dynamics.

\subsection{Summary of discretized equations}

For the sake of clarity, a summary of the different terms required to solve the PBE involving aggregation and breakage, for the continuous and for the 

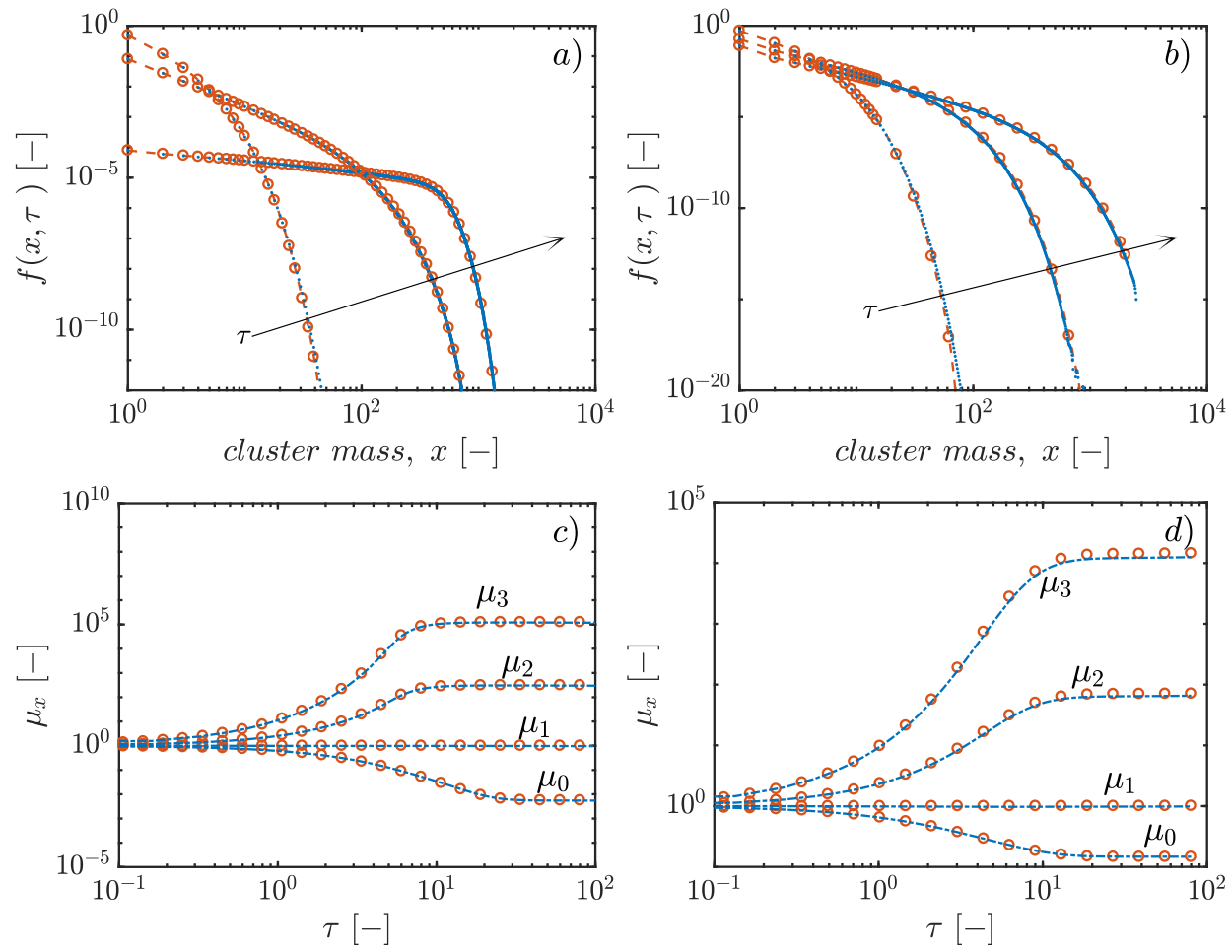

Figure 6: Discrete PBE for aggregation and breakage solved using the mass-dependent breakage (panels a) and c)), and the erosion breakage (panels b) and d)). Distributions are shown for $\tau=\{0.8,4,80\}$, and moments of order $0^{\text {th }}-3^{\text {rd }}$ are reported. Blue dotted lines were obtained using the Gaussian basis functions (GBF) method, the red dashed lines and the circles are the solution of the logarithmic shape-preserving interpolation (LSPI) method. 
discrete cases, is reported in Table 4. In both circumstances, the sum of the four terms is the time derivative $d f\left(x_{i}, t\right) / d t$ at time $t$, which needs to be computed at each time integration step. It can be noticed the similarity between the two formulations, both involving three integrals (one for each grid point of coordinate $x_{i}$ ) to be evaluated by numerical quadrature. In practice, by means of the Euler-Maclaurin approximation, which allows to approximate a series with an integral, the discrete problem is also treated as a pseudo-continuous one. The main difference between the two cases relies on the need, when dealing with discrete problems, to add to each integral the sum of half the values of the integrand functions evaluated at the integration limits, as reported in Table 4. These additional terms are a consequence of the Euler-Maclaurin approximation (cf. Eq.6 and Eq.7) too. Note that, in the integral representing the rate of formation due to aggregation, when the domain of integration is halved (i.e. when integrating up to $x_{i} / 2$ rather than up to $x_{i}-1$ and than halving the result), the correction term for the upper limit of integration needs to be carried out only if $x_{i}$ is odd.

\section{Conclusions}

In the present paper a new numerical approach to solve population balance equations (PBE) has been introduced. The method relies on logarithmic shape-preserving interpolation (LSPI) on finite elements and falls in the socalled "basis functions" category. In the LSPI approach, the PBE are written for specific grid values and the integrals are evaluated by standard numerical quadrature rules. Thus, the resulting system of differential equations is solved without further manipulation. The unknown, off-grid values of the distribution function required for the quadratures are calculated through interpolation from the known values at the grid points using shape-preserving piecewise cubic Hermite polynomials. To increase the efficiency of the interpolation, the logarithm of the distribution is interpolated rather than the distribution itself, given that in many cases distribution functions exhibit exponentially decaying tails.

After providing the theoretical foundation of the LSPI approach, the method has been tested in 8 different scenarios, dealing with aggregation and breakage mechanisms with both continuous and discrete PBE. In all cases the LSPI method performed very well, whether the comparison was made with distributed or averaged properties. Notably, a very small number of differential equations were required in all cases, as low as 3-30, to 


\begin{tabular}{|c|c|c|}
\hline & continuous $f(x, t)$ & discrete $f(x, t)$ \\
\hline domain of $f(x, t)$ & $\begin{array}{l}x_{\min } \leq x \leq x_{\max } \\
\text { with } x \in R \geq 0\end{array}$ & $\begin{array}{l}1 \leq x \leq x_{\max } \\
\text { with } x \in N\end{array}$ \\
\hline $\begin{array}{l}\text { rate of consumption of } \\
\text { aggregates of size } x_{i} \\
\text { due to aggregation }\end{array}$ & $-f\left(x_{i}, t\right) \int_{x_{\min }}^{x_{\max }} m_{i}(y) d y$ & $\begin{array}{l}-f\left(x_{i}, t\right)\left[\frac{m_{i}(1)}{2}\right. \\
\left.\int_{1}^{x_{\max }} m_{i}(y) d y+\frac{m_{i}\left(y_{J}\right)}{2}\right]\end{array}$ \\
\hline $\begin{array}{l}\text { rate of formation of } \\
\text { aggregates of size } x_{i} \\
\text { due to aggregation }\end{array}$ & $\int_{x_{\min }}^{x_{i} / 2} n_{i}(y) d y$ & 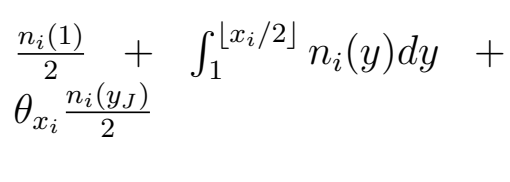 \\
\hline $\begin{array}{l}\text { rate of consumption of } \\
\text { aggregates of size } x_{i} \\
\text { due to breakage }\end{array}$ & $-\gamma\left(x_{i}\right) f\left(x_{i}, t\right)$ & $-\gamma\left(x_{i}\right) f\left(x_{i}, t\right)$ \\
\hline $\begin{array}{l}\text { rate of formation of } \\
\text { aggregates of size } x_{i} \\
\text { due to breakage }\end{array}$ & $\int_{x_{i}}^{x_{\max }} p_{i}(y) d y$ & $\frac{p_{i}(1)}{2}+\int_{x_{i}+1}^{x_{\max }} p_{i}(y) d y+\frac{p_{i}\left(y_{J}\right)}{2}$ \\
\hline \multicolumn{3}{|c|}{ Integrand and auxiliary functions } \\
\hline \multicolumn{3}{|c|}{$m_{i}(y)=\beta\left(x_{i}, y\right) f(y, t)$} \\
\hline \multicolumn{3}{|c|}{$n_{i}(y)=\beta\left(x_{i}-y, y\right) f\left(x_{i}-y, t\right) f(y, t)$} \\
\hline \multicolumn{3}{|c|}{$p_{i}(y)=2 \Gamma\left(x_{i}, y\right) \gamma(y) f(y, t)$} \\
\hline \multicolumn{3}{|c|}{$\theta_{x_{i}}=1$ if $x_{i}$ is odd, $\theta_{x_{i}}=0$ if $x_{i}$ is even } \\
\hline
\end{tabular}

Table 4: Summary of the discretized equations for solving the PBE for the discrete and for the continuous cases. The functions $\beta, \gamma$ and $\Gamma$ represent the aggregation kernel, the breakage kernel and the daughter distribution function, respectively. For the sake of brevity, the explicit time dependency of the integrand functions, $m_{i}, n_{i}$ and $p_{i}$, was omitted. 
cover several order of magnitudes in both coordinates, with a single exception for a particularly stiff aggregation/breakage problem which required 60 grid points. A detailed analysis of the computational efficiency of the method is outside the scope of the present work, but it is worth mentioning that all simulations, carried out with Matlab on a basic desktop PC, required only a few seconds down to fractions of a second, depending upon the problem at hand, demonstrating the efficiency of the method. Undeniably, the computational power evens out many differences among methods, but a significant number of advantages distinguish the LSPI approach from methods of the same category:

- it is easy to apply as no residual needs to be calculated and no manipulation of the original PBE is necessary;

- it provides accurate results with a limited number of finite elements/grid points. This aspect is especially important in view of possible generalizations to multidimensional cases;

- the number of grid points (i.e. of differential equations actually solved) is decoupled from the number of interpolation points used to compute the integrals, allowing to optimize the number of finite elements and the quadrature knots independently.

Overall, several other steps will be necessary to prove the breadth and reliability of the LSPI approach, such as employing it for nucleation and growth problems and solving multidimensional PBE. Nevertheless, this work represents an important step towards the development of efficient interpolationbased strategies to solve PBE.

\section{Acknowledgments}

S.L. gratefully acknowledges the Swiss National Science Foundation (SNSF) for financial support (grant numbers P300P2_167683). 


\section{Appendix - List of symbols}

Table 5: List of Latin symbols

\begin{tabular}{|c|c|c|}
\hline Parameter & Meaning & Units \\
\hline$b_{i}, c_{i}, d_{i}$ & interpolation parameter & - \\
\hline$C$ & parameter in breakage kernel & - \\
\hline$d_{\epsilon}$ & shift factor parameter & - \\
\hline$D_{p}$ & particle diameter & $m$ \\
\hline$E_{\mu}$ & Error on first 4 moments & $\%$ \\
\hline$f(x, t) d x$ & $\begin{array}{l}\text { number concentration of clusters } \\
\text { with mass comprised between } \\
x \text { and } x+\Delta x\end{array}$ & $\# L^{-1}$ \\
\hline$h_{i}$ & length of $i^{\text {th }}$ interval on the grid & - \\
\hline$I$ & number of grid points & \\
\hline & where the PBE are discretized & - \\
\hline$J$ & $\begin{array}{l}\text { number of grid points used } \\
\text { to interpolate } f(x, t) \text { and compute } \\
\text { the integrals of the PBE }\end{array}$ & - \\
\hline$k_{B}$ & Boltzmann constant & $J K^{-1}$ \\
\hline$m_{i}(y), n_{i}(y), p_{i}(y)$ & function used in quadrature & - \\
\hline$P(y, t)$ & sought cubic interpolant & - \\
\hline$N_{0}$ & initial particle concentration & - \\
\hline$s$ & dissociation rate & $s^{-1}$ \\
\hline$t$ & time & $s$ \\
\hline$T$ & temperature & $K$ \\
\hline$x, y$ & internal coordinate of the $\mathrm{PBE}$ & - \\
\hline$x_{0}$ & parameter for analytical solutions & - \\
\hline$x_{i}$ & $\begin{array}{l}i^{t h} \text { grid point where PBEs are solved } \\
\text { where } i=1,2, \ldots, I\end{array}$ & - \\
\hline$\underline{x}$ & vector containing $x_{i}$ & - \\
\hline$x_{\min }, x_{\max }$ & $\begin{array}{l}\text { smallest and largest } \\
\text { cluster considered }\end{array}$ & - \\
\hline$y_{j}$ & $\begin{array}{l}j^{\text {th }} \text { grid point used for quadratures } \\
\text { where } j=1,2, \ldots, J\end{array}$ & - \\
\hline$y$ & vector containing $y_{i}$ & - \\
\hline$\stackrel{\bar{w}}{j}_{j}$ & weights used in quadrature & - \\
\hline$W$ & Fuchs stability factor & - \\
\hline
\end{tabular}


Table 6: List of Greek symbols

\begin{tabular}{lll}
\hline Parameter & Meaning & Units \\
\hline$\beta(x, y)$ & $\begin{array}{l}\text { aggregation rate constant of } \\
\text { an } x \text { - and a } y \text {-sized cluster }\end{array}$ & $\mathrm{m}^{3} \#^{-1} s^{-1}$ \\
$\gamma(x)$ & breakage rate of an $x$-sized cluster & {$\left[\mathrm{s}^{-1}\right]$} \\
$\Gamma(x, y)$ & probability that an $x$-sized cluster & \\
& breaks in position y & {$[-]$} \\
$\delta_{i}$ & first divided difference & - \\
& on the $i^{\text {th } \text { interval }}$ & - \\
$\Delta_{i}$ & segment width for quadratures & - \\
$\epsilon$ & shift factor & $P a s$ \\
$\eta$ & viscosity & - \\
$\theta$ & parameter used for discrete PBE & - \\
$\lambda$ & RLCA kernel parameter & $m^{k} \# L^{-1}$ \\
$\mu_{i}(t)$ & $i^{\text {th }}$ moment of the distribution & \\
$\mu_{i}^{a n}(t)$ & $i^{t h}$ moment of the distribution & $m^{k} \# L^{-1}$ \\
$\tau$ & from analytical solutions & - \\
$\phi$ & non-dimensional time & - \\
$\omega_{1}, \omega_{2}$ & occupied volume fraction & \\
\hline
\end{tabular}

\section{References}

[1] N. Hildebrandt, C. M. Spillmann, W. R. Algar, T. Pons, M. H. Stewart, E. Oh, K. Susumu, S. A. Diaz, J. B. Delehanty, I. L. Medintz, Energy transfer with semiconductor quantum dot bioconjugates: A versatile platform for biosensing, energy harvesting, and other developing applications, Chemical Reviews 117 (2017) 536-711. PMID: 27359326.

[2] I. Lignos, R. Maceiczyk, A. J. deMello, Microfluidic technology: Uncovering the mechanisms of nanocrystal nucleation and growth, Accounts of Chemical Research 50 (2017) 1248-1257. PMID: 28467055.

[3] S. K. S. K. Friedlander, Smoke, dust, and haze : fundamentals of aerosol dynamics, New York : Oxford University Press, 2nd ed edition, 2000. Includes bibliographical references and index.

[4] D. Pfister, G. Storti, F. Tancini, L. Costa, M. Morbidelli, Synthesis and ring-opening polymerization of cyclic butylene 2,5-furandicarboxylate, 
Journal of Macromolecular Chemistry and Physics 216 (2015) 21412146.

[5] L. Nicoud, M. Owczarz, P. Arosio, M. Morbidelli, A multiscale view of therapeutic protein aggregation: A colloid science perspective, Biotechnology Journal 10 (2015) 367-378.

[6] S. Lazzari, L. Nicoud, B. Jaquet, M. Lattuada, M. Morbidelli, Fractallike structures in colloid science, Advances in Colloid and Interface Science 235 (2016) 1-13.

[7] H. Hulburt, S. Katz, Some problems in particle technology, Chemical Engineering Science 19 (1964) 555-574.

[8] D. Ramkrishna, Population balances: Theory and applications to particulate systems in engineering, 2000.

[9] N. Dotson, Polymerization Process Modeling, Advances in interfacial engineering series, VCH, 1996.

[10] L. Costa, G. Storti, M. Morbidelli, L. Ferro, A. Galia, O. Scialdone, G. Filardo, Copolymerization of vdf and hfp in supercritical carbon dioxide: A robust approach for modeling precipitation and dispersion kinetics, Macromolecular Reaction Engineering 6 (2012) 24-44.

[11] F. Teymour, J. D. Campbell, Analysis of the Dynamics of Gelation in Polymerization Reactors Using the "Numerical Fractionation" Technique, Macromolecules 27 (1994) 2460-2469.

[12] Z. Chen, W. Pauer, H.-U. Moritz, J. Prss, H.-J. Warnecke, Modeling of the suspension polymerization process using a particle population balance, Chemical Engineering \& Technology 22 (1999) 609-616.

[13] L. Costa, G. Storti, Kinetic modeling of precipitation and dispersion polymerizations, Advances in Polymer Science - (2017) 1-33.

[14] H. Tobita, A. Hamielec, Modeling of network formation in free radical polymerization, Macromolecules 3105 (1989) 3098-3105.

[15] A. Butté, G. Storti, M. Morbidelli, Evaluation of the Chain Length Distribution in Free-Radical Polymerization, 2. Emulsion Polymerization, Macromolecular Theory and Simulations 11 (2002) 37-52. 
[16] D. Meimaroglou, P. Pladis, A. Baltsas, C. Kiparissides, Prediction of the molecular and polymer solution properties of ldpe in a high-pressure tubular reactor using a novel monte carlo approach, Chemical Engineering Science 66 (2011) 1685 - 1696.

[17] L. Costa, G. Storti, M. Morbidelli, A. Galia, , G. Filardo, The rate of polymerization in two loci reaction systems: Vdf-hfp precipitation copolymerization in supercritical carbon dioxide, Polymer Engineering and Science 51 (2011) 2093-2102.

[18] S. Lazzari, S. Hamzehlou, Y. Reyes, J. R. Leiza, M. R. P. Costa, R. Dias, G. Storti, Bulk crosslinking copolymerization: comparison of different modeling approaches, Macromolecular Reaction Engineering 8 (2014) 678-695.

[19] R. Pokorn, A. Zubov, P. Matuka, F. Lueth, W. Pauer, H.-U. Moritz, J. Kosek, Process model for styrene and n-butyl acrylate emulsion copolymerization in smart-scale tubular reactor, Industrial \& Engineering Chemistry Research 55 (2016) 472-484.

[20] S. Hamzehlou, Y. Reyes, J. R. Leiza, Detailed microstructure investigation of acrylate/methacrylate functional copolymers by kinetic monte carlo simulation, Macromolecular Reaction Engineering 6 (2012) 319329 .

[21] S. Hamzehlou, J. R. Leiza, J. M. Asua, A new approach for mathematical modeling of the dynamic development of particle morphology, Chemical Engineering Journal 304 (2016) 655 - 666.

[22] W. Koch, S. Friedlander, The effect of particle coalescence on the surface area of a coagulating aerosol, Journal of Colloid and Interface Science 140 (1990) $419-427$.

[23] P. T. Spicer, S. E. Pratsinis, Coagulation and fragmentation: Universal steady-state particle-size distribution, AIChE Journal 42 (1996) 16121620 .

[24] P. Sandkühler, J. Sefcik, M. Lattuada, H. Wu, M. Morbidelli, Modeling structure effects on aggregation kinetics in colloidal dispersions, AIChE journal 49 (2003) 1542-1555. 
[25] M. Soos, L. Wang, R. Fox, J. Sefcik, M. Morbidelli, Population balance modeling of aggregation and breakage in turbulent taylorcouette flow, Journal of Colloid and Interface Science 307 (2007) 433 - 446.

[26] M. Kostoglou, Extended cell average technique for the solution of coagulation equation, Journal of Colloid and Interface Science 306 (2007) $72-81$.

[27] C. Kiparissides, A. Alexopoulos, A. Roussos, G. Dompazis, C. Kotoulas, Population balance modeling of particulate polymerization processes, Industrial and Engineering Chemistry Research 43 (2004) 7290-7302.

[28] D. L. Marchisio, R. O. Fox, Solution of population balance equations using the direct quadrature method of moments, Journal of Aerosol Science 36 (2005) $43-73$.

[29] D. Kashchiev, Nucleation, Butterworth-Heinemann, 2000.

[30] J. Schöll, C. Lindenberg, L. Vicum, J. Brozio, M. Mazzotti, Precipitation of $\alpha$ l-glutamic acid: determination of growth kinetics, Faraday Discussions 136 (2007) 247.

[31] M. Iggland, M. Mazzotti, A population balance model for chiral resolution via Viedma ripening, Crystal Growth and Design 11 (2011) 4611-4622.

[32] D. Segets, M. A. Hartig, J. Gradl, W. Peukert, A population balance model of quantum dot formation: Oriented growth and ripening of zno, Chemical engineering science 70 (2012) 4-13.

[33] S. Lazzari, M. Abolhasani, K. F. Jensen, Modeling of the formation kinetics and size distribution evolution of ii-vi quantum dots, Reaction Chemistry \& Engineering (2017).

[34] R. McGraw, S. Nemesure, S. E. Schwartz, Properties and evolution of aerosols with size distributions having identical moments, Journal of Aerosol Science 29 (1998) $761-772$.

[35] D. Marchisio, R. Fox, Computational Models for Polydisperse Particulate and Multiphase Systems, Cambridge Series in Chemical Engineering, Cambridge University Press, 2013. 
[36] L. Costa, U. Trommsdorff, Control strategy and comparison of tuning methods for continuous lactide ring-opening polymerization, Chemical Engineering and Technology 39 (2016) 2117-2125.

[37] M. Asteasuain, C. Sarmoria, A. Brandolin, Recovery of molecular weight distributions from transformed domains. part i. application of pgf to mass balances describing reactions involving free radicals, Polymer 43 (2002) 2513-2527.

[38] A. Brandolin, A. A. Balbueno, M. Asteasuain, Improved numerical inversion methods for the recovery of bivariate distributions of polymer properties from $2 \mathrm{~d}$ probability generating function domains, Computers \& Chemical Engineering 94 (2016) 272-286.

[39] R. Gunawan, I. Fusman, R. D. Braatz, High resolution algorithms for multidimensional population balance equations, AIChE Journal 50 (2004) 2738-2749.

[40] A. Mesbah, H. J. Kramer, A. E. Huesman, P. M. V. den Hof, A control oriented study on the numerical solution of the population balance equation for crystallization processes, Chemical Engineering Science 64 (2009) $4262-4277$.

[41] B. Szilgyi, Z. K. Nagy, Graphical processing unit (gpu) acceleration for numerical solution of population balance models using high resolution finite volume algorithm, Computers \& Chemical Engineering 91 (2016) 167 - 181. 12th International Symposium on Process Systems Engineering \& 25th European Symposium of Computer Aided Process Engineering (PSE-2015/ESCAPE-25), 31 May - 4 June 2015, Copenhagen, Denmark.

[42] S. Kumar, D. Ramkrishna, On the solution of population balance equations by discretization - I. A fixed pivot technique, Chemical Engineering Science 51 (1996) 1311-1332.

[43] M. Nicmanis, M. Hounslow, Finite-element methods for steady-state population balance equations, AICHE JOURNAL 44 (1998) 2258-2272.

[44] M. Vanni, Approximate Population Balance Equations for Aggregation and Breakage Processes, Journal of Colloid and Interface Science 221 (2000) 143-160. 
[45] D. Bertin, I. Cotabarren, J. Pia, V. Bucal, Population balance discretization for growth, attrition, aggregation, breakage and nucleation, Computers \& Chemical Engineering 84 (2016) 132 - 150.

[46] S. Kumar, D. Ramkrishna, On the solution of population balance equations by discretization - II. A moving pivot technique, Chemical Engineering Science 51 (1996) 1333 - 1342.

[47] J. Kumar, Numerical approximations of population balance equations in particulate systems, Ph.D. thesis, Otto-von-Guericke-Universität Magdeburg, Universitätsbibliothek, 2006.

[48] F. Gelbard, J. H. Seinfeld, Numerical solution of the dynamic equation for particulate systems, Journal of Computational Physics 28 (1978) $357-375$.

[49] S. Rigopoulos, A. Jones, Finite-element scheme for solution of the dynamic population balance equation, AICHE JOURNAL 49 (2003) 11271139.

[50] M. Wulkow, Computer aided modeling of polymer reaction engineeringthe status of predici, i-simulation, Macromolecular Reaction Engineering 2 (2008) 461-494.

[51] A. M. Quarteroni, A. Valli, Numerical Approximation of Partial Differential Equations, Springer Publishing Company, Incorporated, 1st ed. 1994. 2nd printing edition, 2008.

[52] I. Kryven, P. D. Iedema, A Novel Approach to Population Balance Modeling of Reactive Polymer Modification Leading to Branching, Macromolecular Theory and Simulations 22 (2013) 89-106.

[53] L. Gardini, A. Servida, M. Morbidelli, S. Carr, Use of orthogonal collocation on finite elements with moving boundaries for fixed bed catalytic reactor simulation, Computers \& Chemical Engineering 9 (1985) 1-17.

[54] J. Solsvik, H. A. Jakobsen, Evaluation of weighted residual methods for the solution of a population balance model describing bubbly flows: The least-squares, Galerkin, tau, and orthogonal collocation methods, Industrial and Engineering Chemistry Research 52 (2013) 15988-16013. 
[55] D. Eyre, C. Wright, G. Reuter, Spline-collocation with adaptive mesh grading for solving stochastic collection equation, Journal of Computational Physics 78 (1988) 288-304.

[56] N. Bellomo, B. Lods, R. Revelli, L. Ridolfi, Generalized Collocation Methods, Modeling and Simulation in Science, Engineering and Technology, Birkhuser, 2008.

[57] F. N. Fritsch, R. E. Carlson, Monotone piecewise cubic interpolation, SIAM Journal on Numerical Analysis 17 (1980) 238-246.

[58] C. Moler, Numerical Computing with Matlab, Society for Industrial and Applied Mathematics, 2004.

[59] N. I. Lebovka, Aggregation of Charged Colloidal Particles, in: Muller, M (Ed.), POLYELECTROLYTE COMPLEXES IN THE DISPERSED AND SOLID STATE I: PRINCIPLES AND THEORY, volume 255 of Advances in Polymer Science, 2014, pp. 57-96.

[60] S. C. Chapra, R. Canale, Numerical Methods for Engineers, McGrawHill, Inc., New York, NY, USA, 5 edition, 2006.

[61] I. Kryven, S. Lazzari, G. Storti, Population Balance Modeling of Aggregation and Coalescence in Colloidal Systems, Macromolecular Theory and Simulations 23 (2014) 170-181.

[62] S. Lazzari, B. Jaquet, L. Colonna, G. Storti, M. Lattuada, M. Morbidelli, Interplay between Aggregation and Coalescence of Polymeric Particles: Experimental and Modeling Insights, Langmuir 31 (2015) 9296-9305.

[63] P. J. Blatz, A. V. Tobolsky, Note on the kinetics of systems manifesting simultaneous polymerization-depolymerization phenomena, The Journal of Physical Chemistry 49 (1945) 77-80. 